\title{
COPD and microalbuminuria: a 12-year follow-up study
}

\author{
Solfrid Romundstad ${ }^{1,2,3}$, Thor Naustdal ${ }^{1,4}$, Pål Richard Romundstad ${ }^{5}$, \\ Hanne Sorger ${ }^{1}$ and Arnulf Langhammer ${ }^{2}$
}

Affiliations: 'Levanger Hospital, Health Trust Nord-Trøndelag, Dept of Internal Medicine, Levanger, ${ }^{2}$ HUNT Research Center, Faculty of Medicine, Norwegian University of Science and Technology (NTNU), Levanger, ${ }^{3}$ Dept of Cancer Research and Molecular Medicine, Faculty of Medicine, Norwegian University of Science and Technology (NTNU), Trondheim, ${ }^{4}$ Norwegian University of Science and Technology (NTNU), Trondheim, and ${ }^{5}$ Dept of Public Health, Faculty of Medicine, Norwegian University of Science and Technology (NTNU), Trondheim, Norway.

Correspondence: S. Romundstad, HUNT Research Center, Faculty of Medicine, NTNU, Forskningsveien 2 , N-7600 Levanger, Norway. E-mail: solfrid.romundstad@intnu.no

ABSTRACT Chronic obstructive pulmonary disease (COPD), low lung function independent of diagnosis and markers of inflammation are all associated with increased morbidity and mortality. Microalbuminuria, reflecting endothelial dysfunction, could be a relevant inflammatory marker of potential systemic effects of COPD. We hypothesised that there was a positive association between microalbuminuria and mortality in individuals with COPD.

We conducted a 12-year follow-up study of 3129 participants in the second survey of the Nord-Trøndelag Health Study (HUNT), Norway. At baseline, albuminuria was analysed in three urine samples and spirometry was performed. Among the participants, 136 had COPD and microalbuminuria, defined as a urinary albumin/creatinine ratio between 2.5 and $30.0 \mathrm{mg} \cdot \mathrm{mmol}^{-1}$. The main outcome measures were hazard ratio of all-cause mortality according to microalbuminuria.

Compared to those with COPD without microalbuminuria, the adjusted hazard ratio for all-cause mortality in those with COPD and microalbuminuria was $1.54,95 \%$ CI 1.16-2.04. This result was similar after excluding cardiovascular disease at baseline. Classifying COPD severity by Global Initiative for Chronic Obstructive Lung Disease, there was a positive association trend with increasing severity stages.

Microalbuminuria is associated with all-cause mortality in individuals with COPD and could be a relevant tool in identification of patients with poor prognosis.

@ERSpublications

Microalbuminuria is associated with mortality in individuals with COPD in this 12-year follow-up study http://ow.ly/rJAw7

For editorial comments see page 951.

Received: Sept 122013 | Accepted after revision: Dec 052013 | First published online: Jan 162014

Support statement: The Microalbuminuria study was financially supported by The Norwegian Health Association, Norway and MSD. The lung study received non-demanding funding from AstraZeneca, Norway.

Conflict of interest: None declared.

Copyright @ERS 2014 


\section{Introduction}

Chronic obstructive pulmonary disease (COPD) is a progressive disease with increasing prevalence $[1,2]$. By 2020, this disease will become the third leading cause of death. It progresses slowly and is associated with an inflammatory reaction and structural changes in small peripheral airways and/or destruction of the lung parenchyma. The disease is currently defined by post-bronchodilator spirometric forced expiratory volume in $1 \mathrm{~s}$ (FEV1)/forced vital capacity $(\mathrm{FVC})<0.70$ [3]. Norwegian studies have shown prevalence in the 20-90 years age group to be $\sim 7 \%$ based on post-bronchodilator spirometry [3].

Patients with COPD often have several comorbidities [4], and many of the comorbidities are due to aging, but common risk factors, such as tobacco smoking, are also important. The atherosclerotic process in COPD is due to inflammation causing dysfunctional endothelium in coronary and cerebral vessels. It is not clear whether there is an overflow of inflammatory mediators predominantly from the lung tissue in COPD patients or if these mediators are independent of the inflammation in the lung [5]. Studies have shown that use of statins (coenzyme A reductase inhibitors) is associated with reduced mortality in COPD patients $[6,7]$, and corresponding results have been found for use of angiotensin-converting enzyme (ACE) inhibitors and angiotensin II blockers in COPD patients [8].

The excretion of small amounts of albumin in the urine, microalbuminuria, has been well documented to predict cardiovascular morbidity and mortality in both diabetic [9] and non-diabetic persons [10-12]. The link between microalbuminuria and atherosclerosis seems to be the endothelial dysfunction with increased permeability and leakage of albumin through the vessel wall [13]. Microalbuminuria is today considered to be the most important renal marker for generalised damage and systemic inflammation in the vascular system [14].

We hypothesised that there was a positive association between microalbuminuria and mortality in individuals with COPD in a large population-based study. Secondly, we wanted to examine the association between microalbuminuria and lung function.

\section{Materials and methods \\ Study subjects}

During 1995-1997, the second survey of the Nord-Trøndelag Health Study (HUNT, Norway), invited all residents $\geqslant 20$ years $(n=93898)$ to take part in the survey and a total of $70 \%$ participated. The survey comprised questionnaires, which included questions on cardiovascular disease (CVD) (angina pectoris, myocardial infarction and stroke), smoking habits, other lifestyle factors, and a clinical examination. Details of the HUNT study design and microalbuminuria screening have been published previously [15, 16]. All those with self-reported diabetes mellitus and/or treated hypertension and a 5\% randomly selected sample of the total population were included in the microalbuminuria screening study. A total of 9598 participants delivered three morning urine samples for microalbuminuria analysis (overall response rate $84 \%$ ). Of these, 3385 had also undergone spirometry as part of participation in the lung study, either selected as included in the random sample or by having reported symptoms of airways obstruction [17]. Those who answered "yes" to one of the questions about urinary tract infection (UTI) in the previous week, persistent haematuria in the previous year or menstruation at the time of urine collection were excluded from the analysis $(\mathrm{n}=118)$. So were 45 individuals with macroalbuminuria (albumin/creatinine ratio (ACR) $\geqslant 30 \mathrm{mg} \cdot \mathrm{mmol}^{-1}$ ) and 93 individuals with missing spirometry values due to unacceptable tests, leaving a total of 3129 subjects included in the main analysis. Figure 1 shows the study population.

All participants signed written, informed consent. All surveys and linkage of data were approved by the Norwegian Data Inspectorate and by the Regional Committee for Medical and Health Research Ethics.

\section{Clinical examination}

The clinical examination included standardised measurement of height, weight, blood pressure and pulse rate. Height was measured without shoes to the nearest centimetre, and weight was measured to the nearest half-kilogram while wearing light clothing without shoes. Three consecutive standardised blood pressure measurements were recorded with $1 \mathrm{~min}$ interval. The measurements were performed in the sitting position, after a minimum of $2 \mathrm{~min}$ rest, using an automatic oscillometric method (Dinamap 845XT; Criticon, Tampa, FL, USA).

\section{Spirometry}

Flow-volume spirometry was recorded with three pneumotachographs (Masterscope Spirometer, version 4.15; Erich Jaeger GmbH, Wuerzburg, Germany) according to the 1994 American Thoracic Society recommendations [18]. The instruments were calibrated twice daily with a $1 \mathrm{~L}$ syringe, and the trained staff performed daily biological controls by assessing their own lung function. All measurements followed a 


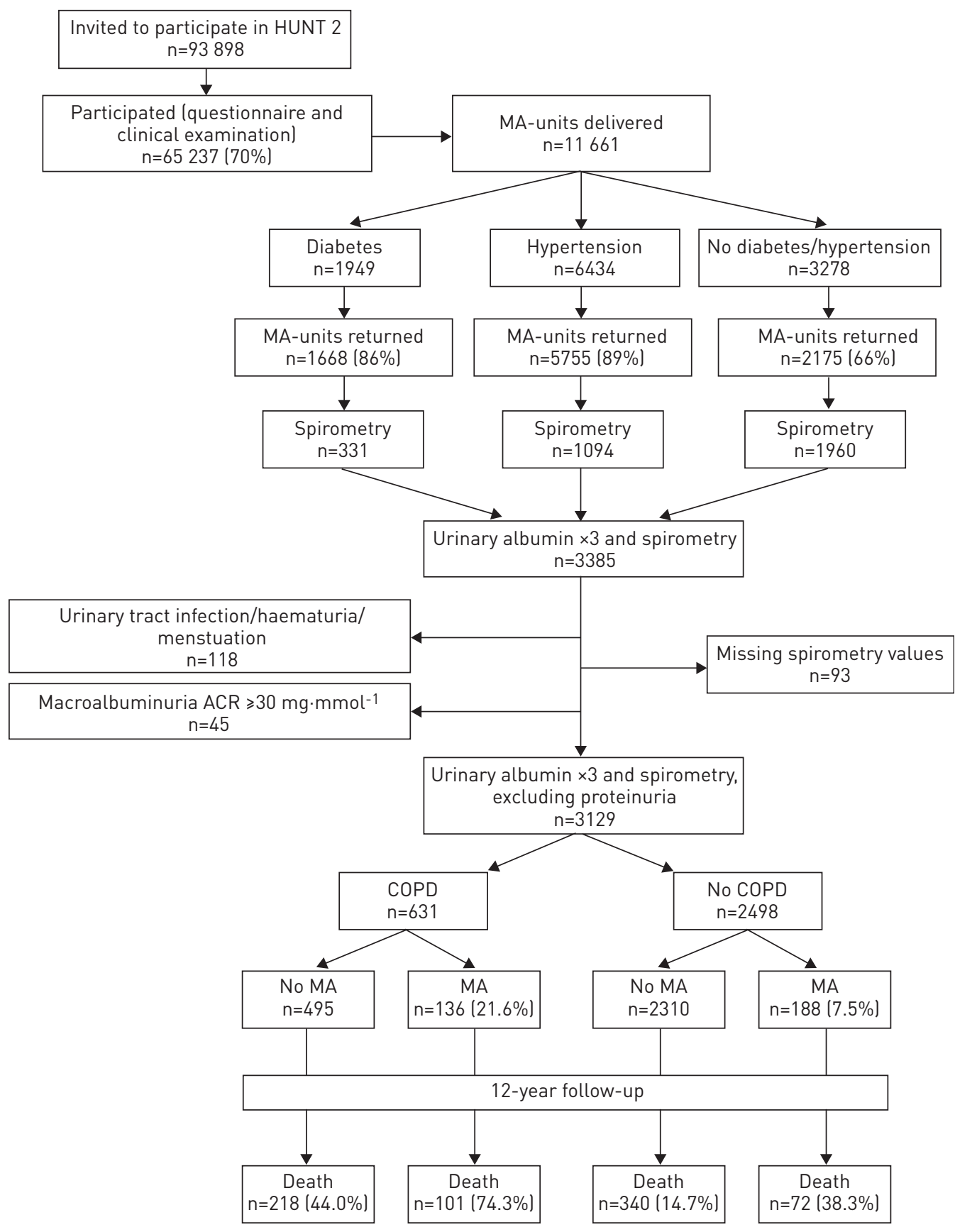

FIGURE 1 Participants in the second Nord-Trøndelag Health Study (HUNT2) and in the microalbuminuria (MA) and spirometry screening at baseline (1995-1997) and after 12-year follow-up. ACR: albumin/creatinine ratio; COPD: chronic obstructive pulmonary disease.

standard protocol, supervised by an experienced clinician. Lung function measurements are given as absolute values and per cent of predicted values according to prediction equations developed from the actual population [19]. In small municipalities, all participants $(n=1108)$ were invited to both pre-and post-bronchodilator spirometry. Because of limited resources, in large municipalities $(n=2021)$, participants with an obstructive pattern of spirometry, defined as FEV1/FVC $<0.75$ or $\mathrm{FEV}_{1}<80 \%$ predicted, were invited to follow-up pre-and post-bronchodilator spirometry. Of those with prebronchodilator spirometry, a total of 1562 (50\%) underwent also post-bronchodilator spirometry. For the present study the pre-bronchodilator results were chosen since the total sample had been tested in this way. However, we performed sensitivity analysis restricted to those with post-bronchodilator test data. 
Urine and blood sampling

The participants included in the microalbuminuria screening received a unit with three plastic receptacles, for three first morning urine samples, and three transport tubes and one envelope for return by mail to the laboratory. Additionally, the participants received instructions for urine collection, information about the microalbuminuria-screening and a questionnaire about UTI, haematuria and menstruation.

Blood sampling was carried out whenever subjects attended the clinic for clinical examination (i.e. in a nonfasting state). Fresh serum and urine samples were analysed at the Central Laboratory at Levanger Hospital, on a Hitachi 91 Autoanalyzer (Hitachi, Mito, Japan). Details of the laboratory methods have been published elsewhere [20]. Urine albumin and creatinine were measured by using an immunoturbidimetric method (anti-human serum albumin; Dako Norway, Oslo, Norway) and Jaffé method, respectively. ACR was used as an expression for urine albumin excretion.

\section{Follow-up and end-points}

The unique 11-digit identification number of every Norwegian citizen enabled individual linkage between the collected information and the Cause of Death Registry, Statistics Norway [21]. Each participant contributed survival time from the date of study entry (date of clinical examination) until the date of death or the end of follow-up on December 31, 2009. Cardiovascular mortality was defined according to International Classification of Diseases (ICD-9 and ICD-10) as follows: hypertensive disease (401-405 and I10-I15), ischaemic heart disease (410-414 and I20-I25), arrhythmia (426-437 and I44-I49), cerebrovascular disease (430-438 and I60-I69) and disease of the arteries (440-447 and I70-I77). Mean follow-up time was 12.0 years.

\section{Statistical analysis}

Body mass index (BMI) was calculated in $\mathrm{kg} \cdot \mathrm{m}^{-2}$. Systolic (SBP) and diastolic blood pressure were included as the mean of the second and third of three measurements. ACR was calculated as urine albumin $\left(\mathrm{mg} \cdot \mathrm{L}^{-1}\right) /$ urine creatinine $\left(\mathrm{mmol} \cdot \mathrm{L}^{-1}\right)$, and was defined as the mean of three ACRs. According to a recently published large meta-analysis concluding that sex-specific cut-off was not necessary to show association between ACR and mortality [22], microalbuminuria was defined as ACR $\geqslant 2.5 \mathrm{mg} \cdot \mathrm{mmol}^{-1}$ and $<30 \mathrm{mg} \cdot \mathrm{mmol}^{-1}$ in both sexes without a sex-dependent correction factor in the formula, which some earlier studies have applied [23-25]. Based on pre-bronchodilator spirometry, COPD was defined by FEV1/FVC $<0.7$ and severity graded as: mild stage I FEV $1 \geqslant 80 \%$ pred; moderate stage II FEV1 $50-79 \%$ pred; and severe/very severe stage III/IV FEV $1<50 \%$ pred, according to the Global Initiative for Chronic Obstructive Lung Disease (GOLD) classification [2]. Since adequate data on symptoms were lacking, we did not use the new GOLD classification. Because few individuals had severe or very severe COPD, these were included as one category. We used the t-test for independent samples, Mann-Whitney U-test and Chi-squared to examine baseline differences between two means or proportions.

The Cox proportional hazards model [26] was used to calculate hazard ratios for all-cause and cardiovascular mortality in subjects with COPD, with and without microalbuminuria. Potential deviation from the proportional hazard assumption was evaluated by log-log plot. Assumptions were met for all models. A priori selected potential confounding factors were used in the multivariable adjusted analysis, including age, sex, diabetes (yes/no), years of education (primary and lower secondary school ( $<10$ years), upper secondary school (10-12 years), and college or university ( $>12$ years)), serum-creatinine, BMI, SBP and treatment of hypertension (yes/no). Since several individuals with antihypertensive treatment were incompletely treated, we also included SBP. The correlation between SBP and treatment of hypertension was low (Pearson correlation coefficient $r=0.33$ ). To reduce residual confounding by underlying disease, we also performed analyses by excluding individuals reporting CVD at baseline $(n=149)$, those with missing data on the questions about CVD $(n=12)$ and those who died in the first year of follow-up $(n=8)$. However, CVD could be an intermediate factor and not a confounder. We also ran analyses adjusting for other inflammatory conditions and long-term illness, like self-reported cancer (yes/no) and other chronic diseases (yes/no). The interactions $(\operatorname{sex} \times \mathrm{ACR})$ and $(\operatorname{sex} \times$ age $)$ were not statistically significant, and the main analyses were performed with the sexes combined to increase the power of the analyses.

Logistic regression analysis was performed to estimate the odds ratio for microalbuminuria according to quintiles of FEV1 \% pred (q5: $>107.0 \%$ pred, q4: $98.1-107.0 \%$ pred, q3: $89.7-98.0 \%$ pred, q2: 78.6-89.6\% pred, q1: $<78.6 \%$ pred). We evaluated a priori selected potential confounding factors in bivariate analysis, and variables significantly associated with microalbuminuria were entered as independent variables in a multivariable analysis. Included confounders were age, sex, SBP, creatinine, treatment of hypertension, selfreported diabetes mellitus and CVD. Test for trend across FEV1 \% pred categories was calculated by treating the categories as ordinal variables in the logistic regression. In the total sample, logistic regression analysis was also performed to estimate odds ratios for all-cause mortality with ACR and FEV1 \% pred as 
continuous independent variables. We adjusted for the same confounders as in Cox analysis in a multivariable model. All statistical analyses were made with IBM SPSS 19.0 (IBM Corporation, Armonk, NY, USA).

\section{Results}

The baseline characteristics showed that those with COPD and microalbuminuria were older, and had higher mean blood pressure, creatinine and glucose levels compared to those without microalbuminuria. They also more frequently had diabetes, treated hypertension and CVD, and there were significantly more deaths during the observation time among those with microalbuminuria. Corresponding differences were found in all GOLD stages, but statistically significant differences were mostly reached in GOLD stage II (table 1). Corresponding differences were also found in those without COPD.

During an observational time of 37704 person-years, 489 males and 342 females died. After adjusting for relevant confounders, those with COPD and microalbuminuria had higher all-cause mortality (hazard ratio 1.54, 95\% CI 1.16-2.04) compared to those with COPD without microalbuminuria (table 2). The cardiovascular mortality was also increased, but did not reach statistical significance (hazard ratio 1.44, 95\% CI 0.93-2.23). When subjects with self-reported CVD at baseline were excluded from the analyses, the association between microalbuminuria and all-cause mortality was not changed (table 2), but the association between microalbuminuria and cardiovascular mortality was further attenuated. Excluding those who died during the first year of follow-up did not change the result. After adjusting for self-reported cancer and "other chronic diseases", neither the estimates nor the significance changed (data not shown).

Using post-bronchodilator FEV1/FVC to define COPD, strengthened the estimates; for all-cause mortality, the multivariable adjusted hazard ratio was 1.72 (95\% CI 1.22-2.43). Exclusion of those with self-reported CVD in those with post-bronchodilator spirometry $(n=288)$ did not change the estimates.

For all GOLD stages of COPD, microalbuminuria was negatively associated with survival, although the associations were not significant (fig. 2). In modified GOLD stage I multivariable adjusted hazard ratio for all-cause mortality was 1.39 (95\% CI 0.69-2.83), in GOLD stage II the hazard ratio was 1.30 (95\% CI 0.90-1.90) and in GOLD stage III/IV the hazard ratio was 1.14 (95\% CI 0.59-2.19) (fig. 2).

In the total sample $(\mathrm{n}=3129)$ the risk for microalbuminuria increased significantly at lower levels of FEV1 $\%$ pred $(\mathrm{p}$ (trend $)<0.001)$. Adjustment for age and sex weakened the association, further adjustments influenced the odds ratio only marginally, and the results were still significant (table 3 ). Sensitivity analysis among 1609 persons with post-bronchodilator FEV1 \% pred showed similar patterns, with a p-trend across the quintiles of $<0.0001$. However, the association was not statistically significant at each quintile except for the lowest quintile (lowest lung function). With quintile q5 (highest FEV1 \% pred) as reference the adjusted estimates were as follows. q4: OR 1.82, 95\% CI 0.93-3.54; q3: OR 1.77, 95\% CI 0.91-3.46; q2: OR 1.44, 95\% CI 0.74-2.80; and q1: OR 3.71, 95\% CI 2.01-6.83.

In the total sample there were statistically significant associations between all-cause mortality and both continuous ACR and FEV1 \% pred, adjusted for each other and multivariable adjusted, OR for ACR of 1.07, 95\% CI 1.03-1.11, and for FEV1 \% pred of 0.06, 95\% CI 0.03-0.11 (table 4).

\section{Discussion}

This study demonstrated a significant positive association between microalbuminuria and all-cause mortality in individuals with COPD. The association was similar after adjusting for several confounders. After dividing into severity groups according to GOLD classification, the association was not significant, but showed a tendency of increased mortality within each stage when ACR was $\geqslant 2.5 \mathrm{mg} \cdot \mathrm{mmol}^{-1}$. We also found an independent association between microalbuminuria and lung function, including all levels of lung function categorised by quintiles.

To our knowledge, this is the first follow-up study demonstrating both the association between microalbuminuria and mortality in individuals with COPD and the association between microalbuminuria and lung function in a population-based sample. A recent publication by CASANOVA et al. [25] showed a high prevalence of microalbuminuria in patients with stable COPD and found that microalbuminuria was associated with hypoxaemia independent of other cardiovascular risk factors. Similar results were found by BULCUN et al. [27], were microalbuminuria was also correlated with the severity of disease. In addition, three small studies have described the prevalence of microalbuminuria in patients with COPD [28-30]. We found a prevalence of $22 \%$ for microalbuminuria, which was quite similar to the $24 \%$ reported by CASANOVA et al. [25]. The other three small studies evaluated the presence of microalbuminuria in COPD during exacerbations and reported higher prevalence. In contrast to the studies where an association between microalbuminuria and hypoxaemia was found, we found an association between microalbuminuria and lung function, which was also 

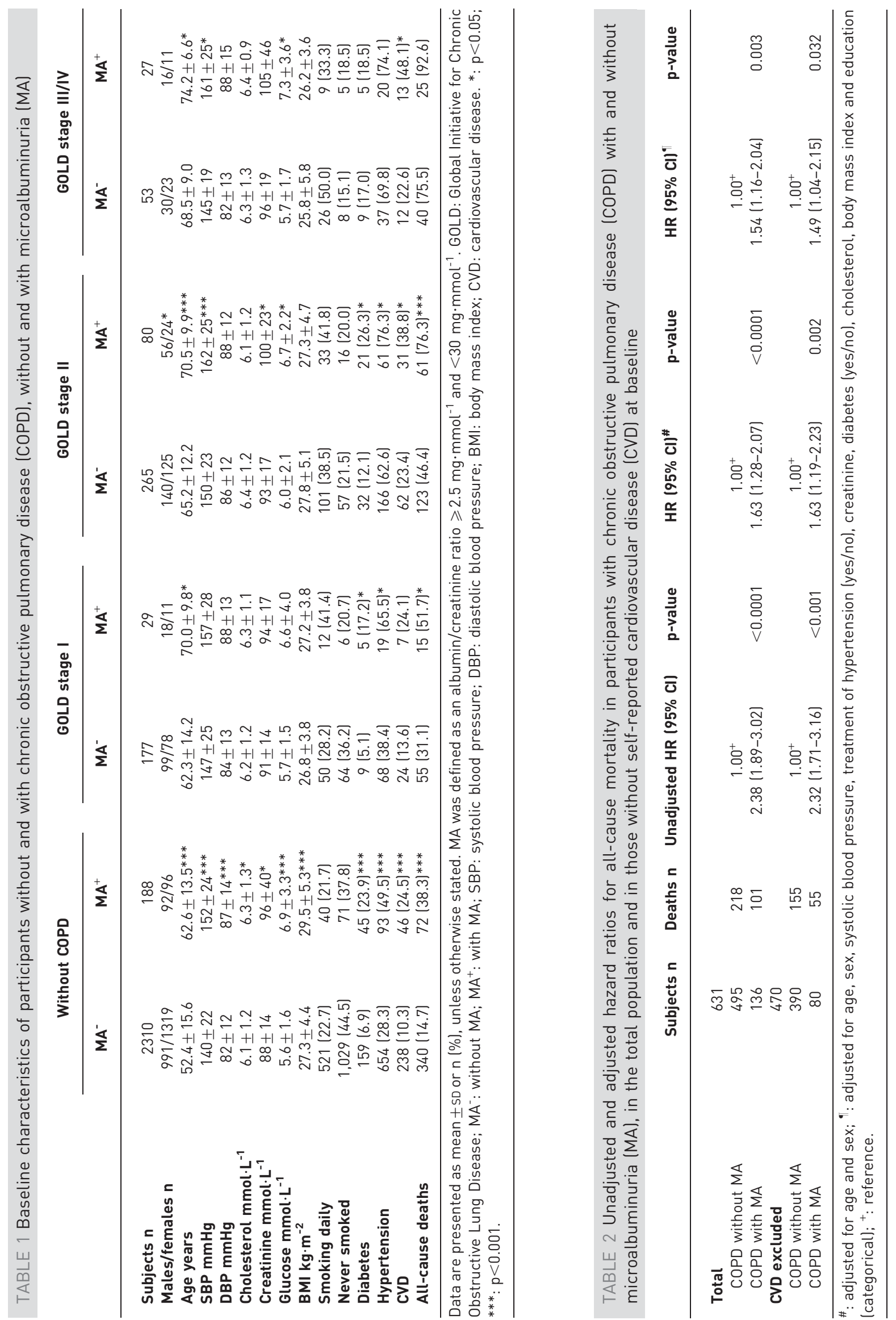


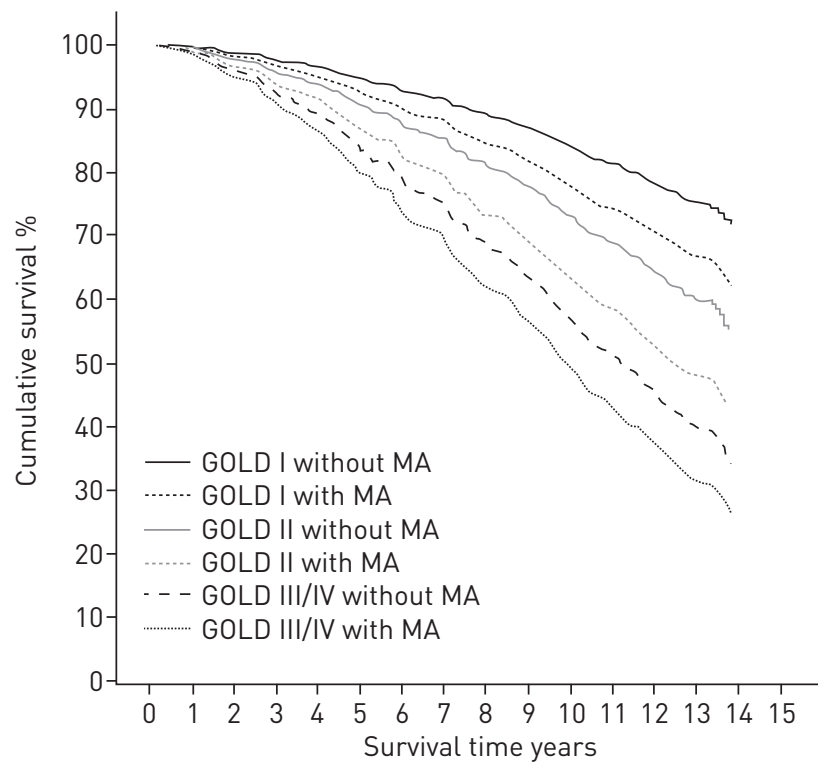

FIGURE 2 Survival plot for all-cause mortality according to severity of airflow limitation, defined by the Global Initiative for Chronic Obstructive Lung Disease (GOLD) classification, and microalbuminuria (MA) showing no significant association. The analyses are adjusted for age, sex, systolic blood pressure, treatment of hypertension, creatinine, diabetes, cholesterol, body mass index and education.

observed after adjustment for several confounders. This might be due to the larger sample size and inclusion of COPD independent of smoking history in the present study.

The population-based approach and the high attendance rate make selection bias less likely. The response rate in the microalbuminuria screening was especially high in the elderly and in those with diabetes and treated hypertension [15]. Another strength of this study was that microalbuminuria analyses were performed on fresh urine samples without long-term storage, in contrast to other studies [11]. Several studies conclude that measuring ACR is a specific and sensitive alternative to 24-h urine collection in population-based microalbuminuria screening [31,32].

One of the limitations of the study includes the use of pre-bronchodilator criteria for diagnosis of COPD. However, sensitivity analyses among $\sim 50 \%$ of participants based on post-bronchodilator tests did not change the estimates substantially. Some of the results were even strengthened, although the power of the analysis decreased. Further, the information about use of medication, which could have influenced microalbuminuria levels and mortality, was limited. However, we adjusted for use or no use of antihypertensive medication, which also includes several cardiovascular medications. At the time of HUNT 2, the use of ACE inhibitors and angiotensin II blockers in Norway were still low. Another limitation includes the diagnosis of UTI. Those who reported UTI in the previous week were excluded from the analyses, but we were not able to adjust for asymptomatic UTI. This could contribute to a non-differential misclassification that might weaken the associations found. Further limitations should be mentioned, like the lack of other inflammatory markers or arterial oxygen tension $\left(\mathrm{PaO}_{2}\right)$ measurements, which was the main factor associated with microalbuminuria in two of the recently published studies [25, 27], and could be an important confounder in the association between microalbuminuria and lung function. However, $\mathrm{PaO}_{2}$ could also be an intermediate step and not necessarily a confounder. The HUNT study is a comprehensive study including many participants and a wider spectrum of diseases. Consequently, more

TABLE 3 Association between lung function, forced expiratory volume in $1 \mathrm{~s}$ (FEV1) and microalbuminuria lalbumin/creatinine ratio $\geqslant 2.5 \mathrm{mg} \cdot \mathrm{mmol}^{-1}$ ) in the total sample

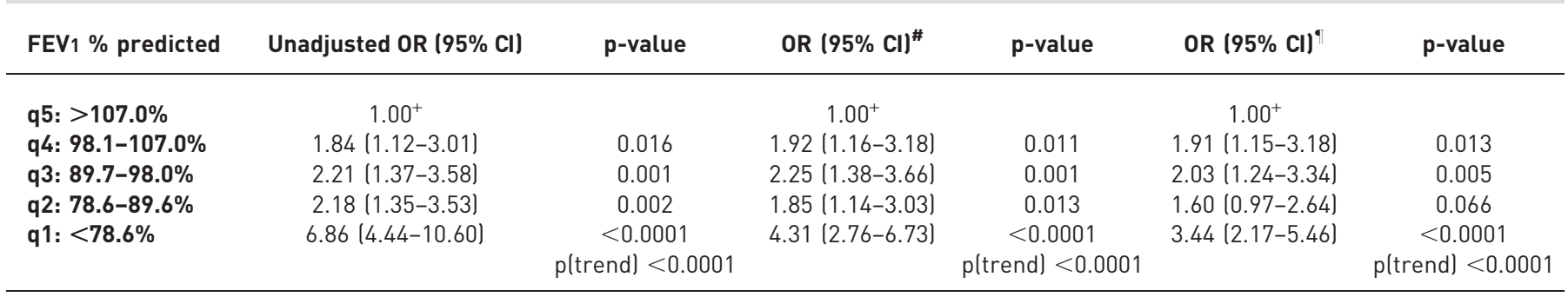

$\mathrm{n}=3129$. q: quintile. ${ }^{\#}$ : adjusted for age and sex; ${ }^{\circledR}$ : adjusted for age, sex, systolic blood pressure, treatment of hypertension (yes/nol, creatinine, diabetes (yes/no) and self-reported cardiovascular disease (yes/no); ${ }^{+}$: reference. 
TABLE 4 Association between lung function, forced expiratory volume in $1 \mathrm{~s}$ (FEV1) (continuous), albumin/creatinine ratio (ACR) (continuous) and all-cause mortality in the total sample

\begin{tabular}{|c|c|c|c|c|c|c|}
\hline & OR $(95 \% \mathrm{CI})^{\#}$ & p-value & OR $(95 \% \mathrm{CI})^{\top}$ & p-value & OR $\left(95 \% \mathrm{CI}^{+}\right.$ & p-value \\
\hline ACR & $1.15(1.11-1.19)$ & $<0.0001$ & $1.08(1.04-1.12)$ & $<0.0001$ & $1.07(1.03-1.11)$ & $<0.0001$ \\
\hline FEV1 \% predicted & $0.02(0.01-0.03)$ & $<0.0001$ & $0.05(0.03-0.09)$ & $<0.0001$ & $0.06(0.03-0.11)$ & $<0.0001$ \\
\hline
\end{tabular}

$\mathrm{n}=3129 .{ }^{\#}$ : ACR and FEV1 \% predicted adjusted for each other; ${ }^{\circ}$ : additionally adjusted for age and sex; ${ }^{+}$: additionally adjusted for age, sex, systolic blood pressure, treatment of hypertension (yes/no), creatinine, diabetes (yes/no), education, cholesterol and body mass index.

specific parameters were not included for most diseases. For the same reasons, measurement of static lung volumes, which could have influenced mortality in COPD patients, were not evaluated in this study. Neither are exacerbations nor pulmonary symptoms assessed. Comorbidities are very heterogeneous, and in this epidemiological study we do not have information about all comorbidities. We have chosen to adjust for the comorbidities and risk factors which are known to be most correlated with microalbuminuria. Finally, variables like haemoglobin A1c and pack-years are not included in the analyses. Both variables have several missing values, and sensitivity analyses with these covariates in Cox and logistic regression models reduced the statistical power substantially, although the estimates changed marginally. As in all observational studies, residual confounding is possible. However, given the strength of the associations observed, we think that the final conclusion would hardly be changed.

An interesting result was the tendency of increased risk of mortality in those with microalbuminuria compared to those without microalbuminuria within the modified GOLD stages. Although the GOLD stage II subgroup was twice as large as the GOLD stage I subgroup, the lack of statistical power might explain why we did not find any significant associations in the GOLD stages.

Although the association with cardiovascular mortality was nonsignificant in this study, the all-cause mortality was still significantly increased after excluding CVD at baseline, which might indicate that microalbuminuria could reflect a systemic inflammation, independent of the atherosclerosis. Testing for microalbuminuria should probably be used more among COPD patient in the clinic. Interventions in microalbuminuria positive individuals with diabetes or hypertension are recommended and should include more aggressive treatment of cardiovascular risk factors like blood pressure, smoking, hyperlipidaemia and overweight [33-35]. So far, there are no recommendations for microalbuminuria screening in otherwise healthy people, although small studies have found a reduction in CVD by intervention with ACE inhibition [36].

In conclusion, microalbuminuria is associated with all-cause mortality in individuals with COPD, and microalbuminuria might be a clinically relevant tool identifying COPD patients with poor prognosis. Whether intervention with more aggressive anti-inflammatory treatment can reduce microalbuminuria and improve the outcome in these subgroups has to be evaluated in further studies.

\section{Acknowledgements}

The Nord-Trøndelag Health Study (The HUNT Study) is a collaboration between the HUNT Research Centre (Faculty of Medicine, Norwegian University of Science and Technology (NTNU), Levanger), The Norwegian Institute of Public Health (Oslo), Nord-Trøndelag County Council, and Levanger Hospital (Levanger). We thank especially the people of Nord-Trøndelag for their endurance and participation.

\section{References}

1 Mannino D, Buist AS. Global burden of COPD: risk factors, prevalence and future trends. Lancet 2009; 370: 765-773.

2 Global Initiative for Chronic Obstructive Lung Disease. Global strategy for the diagnosis, management and prevention of COPD. www.goldcopd.org/guidelines-global-strategy-for-diagnosis-management.html Date last accessed: November 2013. Date last updated: February 2013.

3 Johannessen A, Omenaas ER, Bakke PS, et al. Implications of reversibility testing on prevalence and risk factors for chronic obstructive pulmonary disease: a community study. Thorax 2005; 60: 842-847.

4 Fabbri LM, Luppi F, Beghé B, et al. Complex chronic comorbidities of COPD. Eur Respir J 2008; 31: $204-212$. Barnes PJ, Celli BR. Systemic manifestations and comorbidities of COPD. Eur Respir J 2009; 33: 1165-1185.

6 Søyseth V, Brekke PH, Smith P, et al. Statin use is associated with reduced mortality in COPD. Eur Respir J 2007; 29: 279-283.

7 Marin L, Colombo P, Bebawy M, et al. Chronic obstructive pulmonary disease: patho-physiology, current methods of treatment and the potential for simvastatin in disease management. Expert Opin Drug Deliv 2011; 8: 1205-1220.

8 Mancini GB, Etminan M, Zhang B, et al. Reduction of morbidity and mortality by statins, angiotensin-converting enzyme inhibitors, and angiotensin receptor blockers in patients with chronic obstructive pulmonary disease. J Am Coll Cardiol 2006; 47: 2554-2560. 
Mogensen CE. Microalbuminuria predicts clinical proteinuria and early mortality in maturity-onset diabetes. N Engl J Med 1984; 310: 356-360

10 Gerstein HC, Mann JF, Yi Q, et al. Albuminuria and risk of cardiovascular events, death and heart failure in diabetic and nondiabetic individuals. JAMA 2001; 286: 421-426.

11 Jensen JS, Feldt-Rasmussen B, Strandgaard S, et al. Arterial hypertension, microalbuminuria, and risk of ischemic heart disease. Hypertension 2000; 35: 898-903.

12 Romundstad S, Holmen J, Hallan H, et al. Microalbuminuria and all-cause mortality in treated hypertensive individuals: does sex matter? The Nord-Trøndelag Health Study (HUNT), Norway. Circulation 2003; 108: 2783-2789.

13 Pedrinelli R, Dell'Omo G, Penno G, et al. Non-diabetic, microalbuminuria, endothelial dysfunction and cardiovascular disease. Vasc Med 2001; 6: 257-264.

14 Diercks GF, van Boven AJ, Hillege JL, et al. The importance of microalbuminuria as a cardiovascular risk indicator: a review. Can J Cardiol 2002; 18: 525-535.

15 Hallan H, Romundstad S, Kvenild K, et al. Microalbuminuria in diabetic and hypertensive patients and the general population - consequences of various diagnostic criteria - the Nord-Trøndelag Health Study (HUNT). Scand J Urol Nephrol 2003; 37: 151-158.

16 Krokstad S, Langhammer A, Hveem K, et al. Cohort profile: the HUNT Study, Norway. Int J Epidemiol 2013; 42: 968-977.

17 Langhammer A, Johnsen R, Holmen J, et al. Cigarette smoking gives more respiratory symptoms among women than among men. The Nord-Trøndelag Health Study (HUNT). J Epidemiol Community Health 2000; 54: 917-922.

18 Standardization of spirometry, 1994 update. American Thoracic Society. Am J Respir Crit Care Med 1995; 152: 1107-1136.

19 Langhammer A, Johnsen R, Gulsvik A, et al. Forced spirometry reference values for Norwegian adults: the bronchial obstruction in Nord-Trøndelag Study. Eur Respir J 2001; 18: 770-779.

20 Holmen J, Midthjell K, Krüger Ø, et al. The Nord-Trøndelag Health Study 1995-97 (HUNT 2): Objectives, contents, methods and participation. Norsk Epidemiologi 2003; 13: 19-32.

21 Statistics Norway. Central Bureau of Statistics Kongsvinger, Oslo. www.ssb.no Date last accessed: November 2013.

22 Nitsch D, Grams M, Sang Y, et al. Associations of estimated glomerular filtration rate and albuminuria with mortality and renal failure by sex: a meta-analyses. BMJ 2013; 346: f324.

23 Faulx MD, Storfer-Isser A, Kirchner HL, et al. Obstructive sleep apnea is associated with increased urinary albumin excretion. Sleep 2007; 30: 923-929.

24 Jacobs DR Jr, Murtaugh MA, Steffens M, et al. Gender- and race-specific determination of albumin excretion rate using albumin-to-creatinine ratio in single, untimed urine specimens: the Coronary Artery Risk Development in Young Adults Study. Am J Epidemiol 2002; 155: 1114-1119.

25 Casanova C, de Torres JP, Navarro J, et al. Microalbuminuria and hypoxia in patients with chronic obstructive pulmonary disease. Am J Respir Crit Care Med 2010; 182: 1004-1010.

26 Kleinbaum DG. Survival Analysis: A Self Learning Approach. New York, Springer-Verlag, 1996.

27 Bulcun E, Ekici M, Ekici A, et al. Microalbuminuria in chronic obstructive pulmonary disease. COPD 2013; 10: $186-192$.

28 Kömürcüoğlu A, Kalenci S, Kalenci D, et al. Microalbuminuria in chronic obstructive pulmonary disease. Monaldi Arch Chest Dis 2003; 59: 269-272.

29 Polatli M, Cakir A, Cildag O, et al. Microalbuminuria, von Willebrand factor and fibrinogen levels as markers of the severity in COPD exacerbation. J Thromb Thrombolysis 2008; 26: 97-102.

30 Cogo A, Ciaccia A, Legorini C, et al. Proteinuria in COPD patients with and without respiratory failure. Chest 2003; 123: 652-653.

31 Bakker AJ. Detection of microalbuminuria. Receiver operating characteristic curve analysis favors albumin-tocreatinine ratio over albumin concentration. Diabetes Care 1999; 22: 307-313.

32 Jensen JS, Clausen P, Borch-Johnsen K, et al. Detecting microalbuminuria by urinary albumin/creatinine concentration ratio. Nephrol Dial Transplant 1997; 12: Suppl. 2, 6-9.

33 Summary of the 2007 European Society of Hypertension (ESH) and European Society of Cardiology (ESC) guidelines for the management of arterial hypertension. Vasc Health Risk Manag 2007; 3: 783-795.

34 American Diabetes Association. Executive summary: standards of medical care in diabetes - 2012. Diabetes Care 2012; 35: Suppl. 1, S4-S10

35 Volpe M. Microalbuminuria screening in patients with hypertension: recommendations for clinical practice. Int $J$ Clin Pract 2008; 62: 97-108.

36 Brouwers FP, Asselbergs FW, Hillege HL, et al. Long-term effects of fosinopril and pravastatin on cardiovascular events in subjects with microalbuminuria: ten years of follow-up of Prevention of Renal and Vascular End-stage Disease Intervention Trial (PREVEND IT). Am Heart J 2011; 161: 1171-1178. 